\title{
LA MEATOPLASTIA DORSAL PARA EL TRATAMIENTO DE LA ESTENOSIS DE MEATO EN PACIENTES CON BALANITIS XERÓTICA OBLITERANTE
}

\author{
Pedro Navalón Verdejo 1,2,3, Yoni Pallás Costa', Joaquín Juan Escudero², Milagros Fabuel \\ Deltoro², Felipe Ordoño Domínguez², Elisa Monllor Peidro', Macarena Ramos de Campos² \\ y Francisco Ramada Benlloch?2.
}

'Servicio de Urología. Hospital Casa de Salud. Valencia. España

2 Servicio de Urología. Consorcio Hospital General Universitario. Valencia.

${ }^{3}$ Departamento de Cirugía. Facultad de Medicina. Universidad de Valencia. Valencia. España.

Resumen.- OBJETIVO: Poner de manifiesto nuestra experiencia en el tratamiento de la estenosis de meato secundaria a balanitis xerótica obliterante mediante la técnica de meatoplastia dorsal.

MÉTODOS: Hemos realizado meatoplastia dorsal a 15 pacientes afectos de estenosis de meato balánico secundaria a liquen escleroso y atrófico. Todos los casos fueron intervenidos bajo anestesia local realizando plastia mediante incisión en V invertida según la técnica original de Malone.

RESULTADOS: En todos los pacientes intervenidos se objetivó una mejoría flujométrica así como un excelente resultado estético y escasa incidencia de micción dispersa, existiendo un elevado índice de satisfacción evaluado mediante la realización de un cuestionario. \begin{tabular}{l|}
$\frac{0}{u}$ \\
$\frac{0}{0}$ \\
$\frac{0}{0}$ \\
$\frac{0}{0}$ \\
$\frac{0}{0}$ \\
0 \\
$\frac{0}{0}$ \\
0
\end{tabular}

Pedro Navalón Verdejo

Paseo Alameda 38- 2

46023. Valencia. (España).

pedronavalon@yahoo.com

Trabajo recibido: 15 de mayo 2007
CONCLUSIONES: La meatoplastia dorsal con incisión en $V$ invertida es una técnica de fácil ejecución, que permite ser realizada bajo anestesia local, evita la aparición de hipospadias postoperatorio y proporciona excelentes resultados estéticos y funcionales con un bajo índice de recidivas, por lo que consideramos que debe de incluirse en la cartera de procedimientos habituales del urólogo.

Palabras clave: Pene. Uretra. Balanitis. Liquen escleroso y atrófico.

Summary.- OBJECTIVES: To present our experience in the treatment of meatal stenosis secondary to balanitis xerotica obliterans with the dorsal meatoplasty technique.

METHODS: We have performed dorsal meatoplasty in 15 patients suffering stenosis of the penile meatus secondary to balanitis xerotica obliterans. All cases were performed under local anesthesia, using an inverted $V$ incision plasty following the original of technique from Malone.

RESULTS: All patients showed an improvement in uroflowmetry as well as an excellent aesthetic result, with low incidence of scattered urinary stream; there was a high satisfaction index evaluated by a questionnaire.

CONCLUSIONS: Dorsal meatoplasty with inverted V incision is an easy-to-perform technique, which may be done under local anesthesia, avoids the development of post-operative hypospadias, and achieves excellent aesthetic and functional results with a low recurrence rate; 
therefore, we consider it should be included in the list of frequently performed procedures of the urologist.

Keywords: Penis. Urethra. Balanitis. Sclerous athropic liquen.

\section{INTRODUCCIÓN}

La estenosis de meato uretral puede presentarse tanto en el sexo masculino como en el femenino, pero es mucho más común en el primero. En éstos, a menudo es causada por la inflamación del meato después de la circuncisión, fundamentalmente en niños, constituyendo la entidad conocida como meatitis amoniacal, que conlleva una cicatrización y crecimiento de tejido anormal, generalmente una fina capa de piel que oblitera la abertura uretral (1).

En el adulto, el hallazgo de una estenosis meatal puede estar relacionado con cualquier patología inflamatoria o infecciosa de la uretra (tanto específica como inespecífica), traumatismos (especialmente secundarios a cateterismos permanentes o instrumentación uretral) o bien ser el resultado del fracaso de la reparación previa de un hipospadias. No obstante, la etiología mas frecuente de esta entidad en el paciente adulto la constituye el liquen escleroso (1).

El liquen escleroso, también denominado liquen escleroso y atrófico es una dermatitis crónica, localizada por lo general en la piel del área genital. En los varones, las etapas tardías de esta afección se denominan balanitis xerótica obliterante (2), cuyas lesiones consisten en máculas o placas bien circunscritas de color blanco perlado que pueden afectar al prepucio, glande, meato uretral y fosa navicular. Puede existir descamación hiperqueratósica. La epidermis es a menudo atrófica, con tendencia a resquebrajarse y sufrir lesiones que pueden evolucionar a la ulceración. A medida que la enfermedad se cronifica, la dermis se vuelve esclerótica y se producen cicatrices atróficas (3).

El diagnóstico del liquen escleroso se realiza fundamentalmente sobre bases clínicas, siendo en ocasiones de utilidad la confirmación histopatológica. El diagnóstico diferencial abarca al vitíligo, la hipopigmentación postinflamatoria y el tejido cicatrizal (2).

Cuando sólo está afectado el prepucio, la circuncisión es curativa. Sin embargo, muy frecuentemente se hallan también comprometidos el glande y el meato, en cuyo caso, una combinación de esteroides tópicos y antibióticos puede ayudar a estabilizar la inflamación alrededor del meato. Así, en los pacientes cuyo meato se puede mantener fácilmente en un diámetro que permita un flujo urinario aceptable está justificada la terapia conservadora. No obstante, en los pacientes que presentan una estenosis severa que provoca patología obstructiva, está indicada la corrección quirúrgica.

\section{MATERIAL Y MÉTODOS}

Hemos intervenido a 15 pacientes afectos de estenosis de meato balánico secundaria a balanitis xerótica obliterante realizando meatoplastia dorsal. La edad media fue de 56 años (rango 15 - 82).

Once pacientes $(73 \%)$ habían sido circuncidados con anterioridad, tres de los cuales seguían un programa de autodilataciones periódicas y a otros cuatro $(27 \%)$ se les había realizado previamente una meatotomía ventral.

El seguimiento medio ha sido de 21 meses (rango 2 - 32), realizándose en todos los pacientes un exhaustivo interrogatorio sobre las características del flujo urinario así como una minuciosa exploración clínica abriendo el neomeato para visualizar la uretra. Asimismo, el paciente rellena un breve cuestionario acerca del grado de satisfacción con los resultados de la intervención.

Todos los pacientes fueron intervenidos bajo anestesia local, realizando una meatoplastia dorsal mediante la siguiente secuencia operatoria:

1.- Aplicación por el paciente en su domicilio, aproximadamente una hora antes de la intervención, de una crema de lidocaína y prilocaína al 50\% (crema EMLA), extendida tópicamente sobre toda la superficie del pene.

2.- Infiltración troncular en la base del pene con anestésico local (lidocaína $2 \%$ )

3.- Opcionalmente se puede colocar un torniquete en la base del pene para minimizar la hemorragia y facilitar la intervención, aunque últimamente prescindimos de esta maniobra.

4.- Colocación de 2 suturas de referencia de nylon 4/0 a cada lado del meato estenótico (Figura 1-A).

5.- Mínima incisión del meato en su cara ventral y otra algo más extensa en su cara dorsal (Figura 1-B) con posterior sutura discontinua de poligalactina $4 / 0$ 

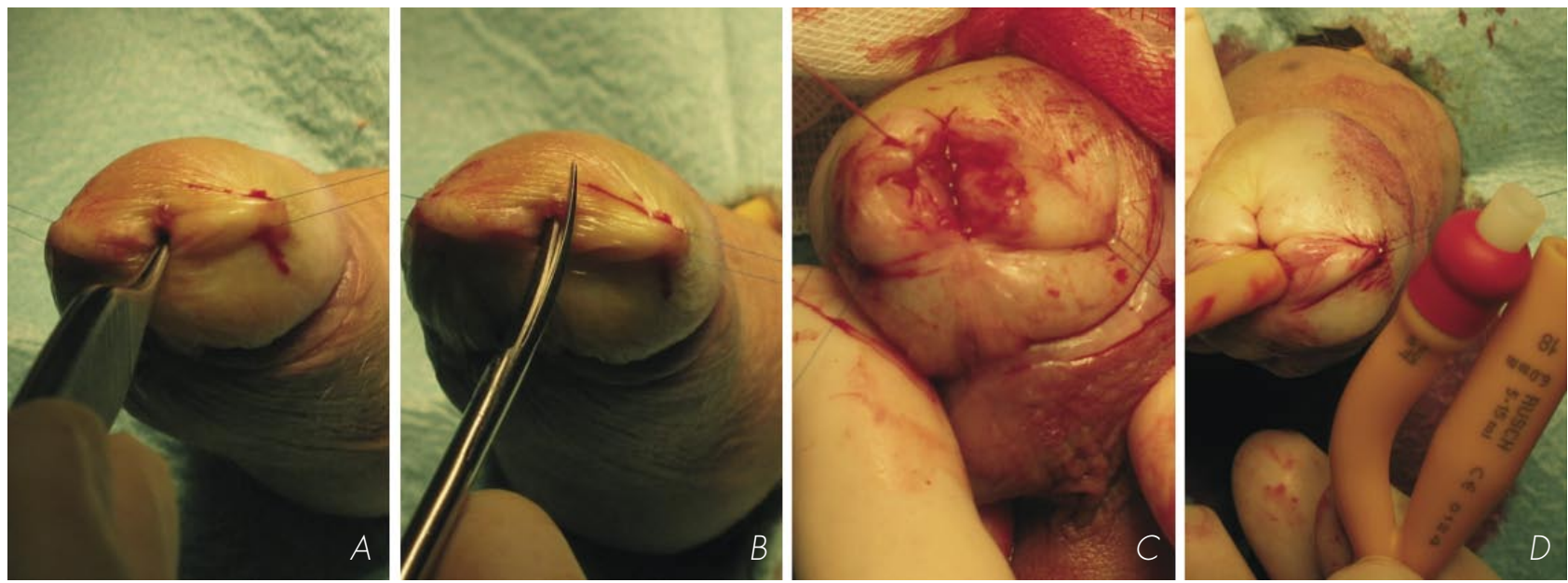

FIGURA 1. Secuencia operatoria de la meatotomía y comprobación de la indemnidad de la uretra proximal.

a 6/0 oponiendo el epitelio uretral al del glande. Esta maniobra ensancha el meato, pero le confiere un aspecto fruncido antiestético (Figura 1-C).

6.- Comprobación de la indemnidad de la uretra proximal mediante la introducción de una pinza o de unos pocos centímetros de sonda 18 ó $20 \mathrm{Ch}$. (Figura 1-D). En el caso que la estenosis se extendiera de forma significativa en la fosa navicular, la técnica descrita fracasaría, por lo que sería necesario realizar otro tipo de uretroplastia.

7.- Incisión relajante en glande en forma de $\mathrm{V}$ inverti$\mathrm{da}$, con el ápex de la $\mathrm{V}$ cerca del límite proximal de la meatotomía dorsal (Figura 2-A).

8.- Liberación de los bordes de la incisión en $V$ mediante disección aguda.

9.- Sutura continua con poligalactina $5 / 0$ a $7 / 0$ oponiendo los bordes internos derecho e izquierdo de la incisión en $\mathrm{V}$, formando el techo de la neouretra (Figura 2-B y $2-C$ ).

10.- Sutura con puntos sueltos de poligalactina $4 / 0$ a $6 / 0$ de los bordes externos de la incisión en $V$, dando como resultado un meato de morfología rasgada, pero con buen calibre (Figura 2-D y 2-E).

\section{RESULTADOS}

Todos los pacientes fueron intervenidos bajo anestesia local y en régimen ambulatorio estricto, no precisándose en ningún caso reconversión de la técnica quirúrgica ni utilización de sedoanalgesia adicional.

No hemos evidenciado recurrencia de la estenosis en ninguno de los casos durante un tiempo de seguimiento medio de 21 meses, obteniendo en todos ellos una recuperación tanto subjetiva como ob-
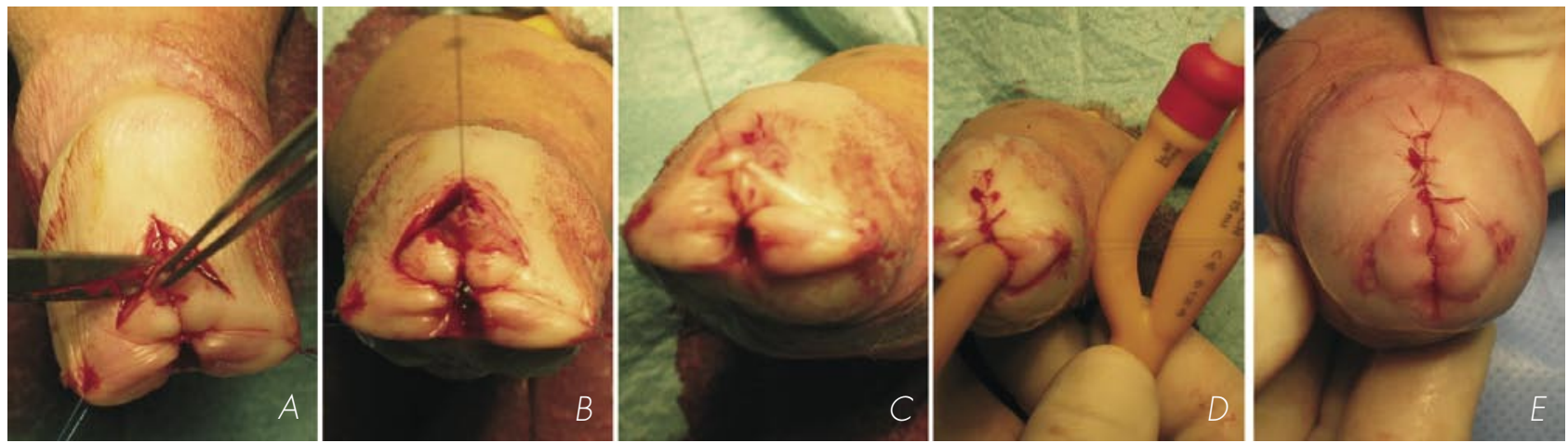

FIGURA 2. Secuencia operatoria de la meatoplastia y resultados en el postoperatorio inmediato. 


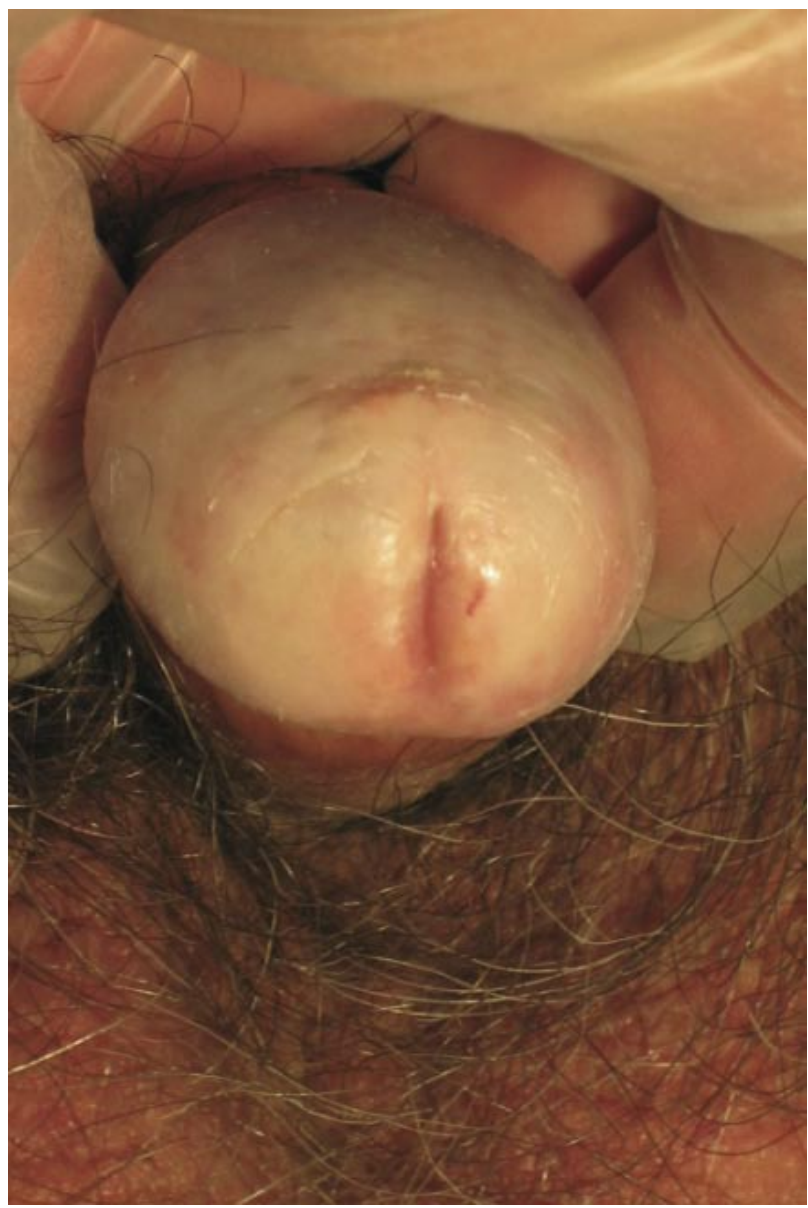

FIGURA 3. Resultado postoperatorio a largo plazo.

jetiva del flujo miccional, habiéndose observado una mejoría significativa en la evaluación flujométrica, siendo el pico de flujo medio preoperatorio de 7,2 \pm $2,9 \mathrm{cc} / \mathrm{s}$ y de $16,4+3,7 \mathrm{cc} / \mathrm{s}$ en el postoperatorio. Asimismo, la mayoría de los pacientes no refirieron dispersión del chorro miccional, y únicamente 3 de ellos $(20 \%)$ manifestaron presentar esta molesta condición, tan frecuente con otros procedimientos.

Todos los pacientes contestaron el cuestionario sobre el grado de satisfacción con el aspecto estético obtenido con la intervención, cuyos resultados se detallan en la Tabla I.

\section{DISCUSIÓN}

Son múltiples las técnicas que se han descrito para el tratamiento de las complicaciones uretrales del liquen escleroso, fundamentalmente las que utilizan piel extragenital o mucosa bucal destinadas a corregir las lesiones que se extienden a la fosa navi- cular (4). No obstante, existen muy pocos artículos que hagan referencia al tratamiento de las lesiones puras del meato, las cuales, aunque constituyan una patología menos importante por su menor extensión en la uretra, son mucho más frecuentes que las primeras. Igualmente, aunque la estenosis meatal en el contexto del liquen escleroso constituye una condición menos severa que cuando la enfermedad afecta a la uretra, esto no implica que represente una entidad menos peligrosa, pues aunque raros, se han descrito casos de pacientes con fracaso renal secundario a la obstrucción crónica provocada por esta patología (5).

A la hora de realizar una meatoplastia, el urólogo no sólo debe considerar los aspectos funcionales sino también los estéticos. Así, una meatoplastia ideal además de resolver la obstrucción de forma permanente, debe proporcionar un meato de morfología rasgada en la punta del glande que permita una emisión de orina sin provocar dispersión del chorro miccional.

Históricamente se han descrito diversos procedimientos destinados a resolver la estenosis meatal, incluyendo la dilatación del meato, la meatotomía ventral simple y varios tipos de uretroplastias distales, e incluso fotovaporización circunferencial del meato con láser (6), pero la realidad es que ninguno de estos procedimientos cumple todos los criterios de la meatoplastia ideal anteriomente expuestos, particularmente cuando se trata de una estenosis severa o cuando el meato tiene una localización ventral.

En pacientes con balanitis xerótica, la dilatación del meato no suele ser efectiva, pues la recidiva precoz es la norma. Así tres de nuestros pacientes se realizaban autodilataciones periódicas frecuentes. Asimismo, la meatotomía simple puede ser efectiva, aunque presentan un elevado índice de recurrencias

\section{TABLA I. SATISFACCIÓN CON EL RESULTADO ESTÉTICO OBTENIDO.}

\begin{tabular}{|lcc|}
\hline CALIFICACIÓN & № Casos & $\%$ \\
\hline Excelente & 10 & $67 \%$ \\
\hline Bueno & 4 & $27 \%$ \\
\hline Regular & 1 & $7 \%$ \\
\hline Deficiente & 0 & $0 \%$ \\
\hline
\end{tabular}


$(7,8)$. Igualmente, la meatoplastia con colgajo cutáneo ventral tipo Blandy (9), aunque disminuye la probabilidad de recidiva de la estrechez, proporciona unos resultados estéticos muy poco satisfactorios, particularmente cuando el meato presenta una localización ventral, dando lugar su corrección a un meato hipospádico. La modificación realizada posteriormente por De Sy (10), puede mejorar el aspecto cosmético, pero como el abordaje también es ventral, la convierte en una técnica poco adecuada para un meato ventralmente localizado.

La ventaja de esta nueva técnica de Malone es que como realiza tanto meatotomía dorsal como ventral, proporciona una excelente apertura del meato, presentando una menor tendencia a la reestenosis. También conserva el meato en la punta del glande, ya que una incisión de tamaño semejante realizada sólo en la cara ventral daría lugar a un meato hipospádico. Asimismo, junto a unos excelentes resultados estéticos (Figura 3), proporciona una escasa incidencia de micción dispersa, que en nuestra experiencia constituye una frecuente causa de queja de los pacientes intervenidos con otras técnicas.

\section{CONCLUSIÓN}

La meatoplastia dorsal con incisión en $\mathrm{V}$ invertida es una técnica de fácil ejecución, que permite ser realizada bajo anestesia local, evita la aparición de hipospadias postoperatorio y proporciona excelentes resultados estéticos y funcionales con un bajo índice de recidivas, por lo que consideramos que debe de incluirse en la cartera de procedimientos habituales del urólogo.

\section{BIBLIOGRAFÍA y LECTURAS RECOMENDADAS (*lectura de interés $y^{* *}$ lectura fundamental)}

*1. JORDAN, G.H.; SCHLOSSBERG, S.M.: "Cirugía del pene y la uretra". Walls, P.C. Campbell Urología $8^{a}$ Edición. Pág. 4253-4324, Ed. Panamericana, Madrid, 2004.

2. MARGOLIS, D.J.: "Enfermedades cutáneas de los genitales externos". Walls, P.C. Campbell Urología $8^{a}$ Edición. Pág. 777-794, Ed. Panamericana, Madrid, 2004.

3. VIRASORO, R.; KAHN, A.G.; SECIN, F.P.: "Balanitis xerótica obliterante". Rev. Arg. de Urol., 68: 125, 2003.

4. VENN, S.N.; MUNDY, A.R.: "Urethroplasty for balanitis xerotica obliterans". Br. J. Urol., 81: 735, 1998.

**5. MALONE, P.: "A new technique for meatal stenosis in patients with lichen sclerosus". J. Urol., 172: 949, 2004.

6. HREBINCO, R.L.: "Circumferential laser vaporization for severe meatal stenosis secondary to balanitis xerotica obliterans". J. Urol., 156: 1735, 1966.

7. UPADHYAY, V.; HAMMODAT, H.M.; PEASE, P.W.: "Post circumcision meatal stenosis: 12 years'experience". N. Z. Med. J., 111: 57, 1998.

8. EL-KASABAY, A.W.; EL-BAZ, M.A.; EL-ZAYAT, T.: "Eversion meatoplasty in management of urethral meatal stenosis". Int. Urol. Nephrol., 28: 229, 1996.

9. BLANDY, J.P.; TRESIDDER, G.C.: "Meatoplasty". Br. J. Urol., 39: 633, 1967.

10. DE SY, W.A.: "Aesthetic repair of meatal stricture”. J. Urol., 132: 678, 1984. 